\title{
Genistein reduced the neural apoptosis in the brain of ovariectomised rats by modulating mitochondrial oxidative stress
}

\author{
Yan-Hong Huang ${ }^{1}$ and Qing-Hong Zhang ${ }^{2 *}$ \\ ${ }^{1}$ Department of Obstetrics and Gynecology, Xi'jing Hospital, 4th Military Medical University, 17 Changle Westroad, \\ $X i$ 'an 710032, People's Republic of China \\ ${ }^{2}$ Department of Microbiology and Immunology, Burns Institute, First, Hospital Affiliated to Chinese PLA General Hospital, \\ Room 301, 51 Fucheng Road, Haidian, Beijing 100037, People's Republic of China
}

(Received 19 January 2010 - Revised 16 April 2010 - Accepted 22 April 2010 - First published online 28 June 2010)

The present study was undertaken to investigate the antioxidant effect of chronic ingestion of genistein (Gen) against neural death in the brain of ovariectomised (Ovx) rats. The rats were randomly divided into five groups, i.e. sham-operated (sham), Ovx-only, Ovx with $17 \beta$-oestradiol, Ovx with low $(15 \mathrm{mg} / \mathrm{kg})$ and high $(30 \mathrm{mg} / \mathrm{kg})$ doses of Gen (Gen-L and Gen-H), and were orally administered daily with drugs or vehicle for 6 weeks The learning and memory abilities were measured by Morris water maze test. Oxidative damages in the brain were evaluated by the level of superoxide dismutase (SOD), malondialdehyde (MDA) and monoamine oxidase (MAO) activities. Neural apoptosis was shown by terminal deoxynucleotidyl transferase dUTP nick end labelling (TUNEL) staining and caspase-3 activity. In the visual learning and memory test, there were no significant differences among the population means of the five groups. While in the probe trial test, the Gen-L group instead of the Gen- $\mathrm{H}$ group exhibited reduced escape latency and increased memory frequency than the Ovx group. Although both doses of Gen could reduce acetylcholinesterase activity, only a low dose of Gen could diminish MDA activity significantly in frontal cortex and enhance SOD content in the hippocampus. In contrast, MAO content was decreased in the cortex by either dose of Gen, while in the hippocampus, only a high dose of Gen appeared to be effective. Interestingly, Gen at both the doses could attenuate the increased number of TUNEL-positive neurons and caspase-3 activity in Ovx rats. These results suggest that Gen confers protection against Ovx-induced neurodegeneration by attenuating oxidative stress, lipid peroxidation and the mitochondria-mediated apoptotic pathway in a region- and dose-dependent manner.

Genistein: Menopause: Brain: Oxidative stress

Mitochondria have become a primary focus for the regulation of viability and death of neurons. As a major source of reactive oxygen species and oxidative stress, mitochondrial dysfunction has been linked to numerous diseases including those of the nervous system ${ }^{(1)}$.

The key contributors to the neurodegenerative disorders include superoxide dismutase (SOD), monoamine oxidases (MAO) and malondialdehyde (MDA). SOD dismutates superoxide to hydrogen peroxide, and overexpression of SOD leads to an increased production of hydrogen peroxide and may lead to brain injury. Large amounts of unsaturated lipids are also present in the brain which are easy targets for free radicals. The resulting lipid peroxidation initiates a series of complex, autocatalytic propagation reactions that generate a variety of thiobarbituric acid-reactive substances such as the MDA, indicating the oxidative damage in the brain $^{(2)}$. MAO are mitochondria-bound isoenzymes that catalyse the oxidative deamination. The byproducts of MAO-mediated reactions include several chemical species with neurotoxic potential. So, the prolonged excessive activity of these enzymes may be conducive to mitochondrial damage and neurodegenerative disturbances ${ }^{(3)}$.
It is intriguing that the vulnerable component of mitochondrial function is ovarian controlled in the central nervous system $^{(4)}$. It was reported that oestrogen could increase the activity, but not the levels, of MnSOD in the brain. Oxidative stress damage caused by chronic exposure to ozone could be prevented by oestradiol treatment ${ }^{(5)}$. Thus, oestrogen suppression of mitochondrial oxidative stress may influence neurodegenerative disorders in ageing women ${ }^{(6)}$. As such, oestrogens and novel non-hormonal analogues have come to figure prominently in the protection against neurodegeneration contributed by reduced oestrogen levels after menopause ${ }^{(7)}$.

Genistein (Gen) has been shown to have the therapeutic potential to reduce cognitive decline and neurodegenerative disease associated with menopause either alone ${ }^{(8)}$ or in combination with other phyto-oestrogens ${ }^{(9)}$. However, the mechanism of its neuroprotective effect remains unclear. Recently, it was found that Gen could diminish oxidative damage in the brain. In vitro, Gen at $0.5 \mu \mathrm{M}$ could mimic the protective effect of oestradiol by decreasing the rate of reactive oxygen species formation and preventing the release of cytochrome $c$ from the mitochondria ${ }^{(10)}$. In vivo, soya consumption could

Abbreviations: AchE, acetylcholinesterase; E2, 17ß-oestradiol; Gen, genistein; Gen-H, Gen-high; Gen-L, Gen-low; MAO, monoamine oxidases; MDA, malondialdehyde; Ovx, ovariectomised; SOD, superoxide dismutase; TUNEL, terminal deoxynucleotidyl transferase dUTP nick end labelling.

* Corresponding author: Dr Q.-H. Zhang, fax +86010 68989955, email z_qinghong@yahoo.com.cn 
reduce plasma MDA and increase plasma total antioxidant capacity in postmenopausal women ${ }^{(11)}$. A pioneer study specified that Gen $(2.5 \mathrm{mg} / \mathrm{rat})$ that is injected subcutaneously every other day for $30 \mathrm{~d}$ could increase serum SOD and reduce MDA levels in rats ${ }^{(8)}$. Although serum parameters could reflect the overall oxidative status to some extent, the direct effect of Gen on the brain remains to be explored.

Our purpose was to investigate the antioxidative mechanism underlying the neuroprotective effects of Gen. Consequences on learning and memory were subsequently investigated using Morris water maze test. The acetylcholinesterase (AchE) activity, which is essential for the normal functioning of brain, was also examined. The oxidative stress status was revealed by detecting SOD and MDA contents and MAO activity in the brain. Moreover, neural apoptosis was revealed by terminal deoxynucleotidyl transferase dUTP nick end labelling (TUNEL) assay and caspase-3 activity.

\section{Experimental methods}

\section{Experimental animals and drug administration}

Sixty-five female Sprague-Dawley rats (180-220g, 3-4 months old), provided by the Animal Facility of 4th Military Medical University, were housed in a temperature-controlled room $\left(22-24^{\circ} \mathrm{C}\right)$ with water and food freely available. The study followed the national guidelines for care and use of animals, and all the procedures were approved by the Ethics Committee for the Use of Experimental Animals. The rats were randomly divided into five groups, i.e. shamoperated (sham), ovariectomised (Ovx)-only, Ovx with $17 \beta$-oestradiol (E2), Ovx with low and high doses of Gen (Gen-L and Gen-H). All the rats were anesthetised with sodium pentobarbital for surgery.

One week after the surgery, the rats were orally administered daily with drugs or vehicles for six consecutive weeks. Gen (Sigma, St Louis, MO, USA) was dissolved in sesame oil and injected at $15 \mathrm{mg} / \mathrm{kg}$ for the Gen-L group and at $30 \mathrm{mg} / \mathrm{kg}$ for the Gen-H group, respectively, which was documented to confer significant neuroprotection ${ }^{(8)}$. E2 (Sigma) was chosen at the dose of $100 \mu \mathrm{g} / \mathrm{kg}$ as reported ${ }^{(12)}$. The sham and Ovx rats received sesame oil as the vehicle control. Eight rats from each group were used for behavioural test and histological study, and another five rats from each group were used for the biochemical measurement.

\section{Behavioural test}

Behavioural test began on day 43 after surgery and lasted for $5 \mathrm{~d}$. The process included two tests. First, place navigation was performed. Daily sessions consisted of two trials separated by a 90 min interval. Trials began with the rat placed in the pool facing the side wall at the start position and ended once the rat had found the platform. If the rat had not found the platform within $120 \mathrm{~s}$, it was guided there by hand. After a period of $30 \mathrm{~s}$ on the platform, the rat was immediately replaced in the pool at a different start position for the next trial. The escape latency, i.e. the time of locating the hidden platform, was automatically calculated by a commercial video/computer system (Jieri'ou Limited, Beijing, China). Secondly, a spatial probe trial was performed during the afternoon of the last day.
For this, the platform was removed, and the time spent in the region that previously contained the platform, called 'frequency of memory', and the time taken to reach the platform location, called 'swimming time', were recorded over $120 \mathrm{~s}$.

\section{Preparation of tissue extracts}

The extracts of brain cortex and hippocampus of five rats were prepared by the following procedure. Tissues were weighed and homogenised in saline on ice at a concentration of $10 \mathrm{~g} / \mathrm{l}$. Then, the homogenates were centrifuged at $4000 \mathrm{rpm}$ for $20 \mathrm{~min}$ at $4^{\circ} \mathrm{C}$, and the supernatants were collected and stored at $-70^{\circ} \mathrm{C}$.

\section{Biochemical assay}

The biochemical analysis was performed as per instructions (Jian Cheng Limited, NanJing, China). AchE activity was determined colorimetrically by a microtiter plate-adapted modification of the Ellman method. The initial absorbance and the absorbance after $4 \mathrm{~min}$ were read at $412 \mathrm{~nm}$. Brain SOD was measured by the xanthine oxidase-nitroblue tetrazolium assay. One unit of SOD is defined as the amount required to inhibit the reduction rate of nitroblue tetrazolium by $50 \%$. The results were expressed as specific activity of the enzyme in units per $\mathrm{mg}$ protein (U/mg). MDA was measured by thiobarbituric acid test. Briefly, an aliquot $(100 \mu \mathrm{l})$ of the supernatant was added to the reaction mixture containing $100 \mu \mathrm{l}$ of $8.1 \%$ SDS, $750 \mu \mathrm{l}$ of $20 \%$ acetic acid $(\mathrm{pH} 3.5), 750 \mu \mathrm{l}$ of $0.8 \%$ thiobarbituric acid and $300 \mu \mathrm{l}$ of distilled water. Samples were then boiled for $1 \mathrm{~h}$ at $95^{\circ} \mathrm{C}$ and centrifuged at $4000 \mathrm{~g}$ for $10 \mathrm{~min}$. The absorbance of the supernatant was measured spectrophotometrically at $532 \mathrm{~nm}$, and the results were expressed as nmol per $\mathrm{mg}$ of protein. The level of MAO activity, as measured by ammonia production, was detected as per instructions, and was expressed as nmol per mg of protein.

The amount of soluble protein was determined as described by the method of Bradford.

\section{Apoptosis examination}

Measurement of caspase- 3 activity (Clontech, Mountain View, CA, USA) was done as described by Enari et al. ${ }^{(13)}$. Briefly, cytosolic extract $(100 \mu \mathrm{g}$ protein) was incubated for $1 \mathrm{~h}$ at $37^{\circ} \mathrm{C}$ with the reaction buffer $(25 \mathrm{mM}$-HEPES, $\mathrm{pH} 7 \cdot 5,10 \%$ sucrose, $\quad 0.1 \%$ 3-[(3-cholamidopropyl)dimethylammonio]1-propanesulfonic acid (CHAPS), $5 \mathrm{~mm}$-dithiothreitol and $5 \mathrm{mM}$-EDTA) in a total volume of $150 \mu \mathrm{l}$ containing $25 \mu \mathrm{M}$ acetyl-asp-glu-val-asp-p-nitroanilide (Sigma). Enzymecatalysed release of $p$-nitroanilide was measured at $405 \mathrm{~nm}$ using a microtiter plate reader.

TUNEL staining was performed using in situ cell death detection kit (Promega, Beijing, China) on the cryostat coronal sections as described. The number of TUNEL-positive cells was counted by an investigator who was blinded to the experimental conditions. Three fields $\left(1000 \mu \mathrm{m}^{2} /\right.$ field $)$ in one section were randomly chosen, and five sections were counted in one group. The apoptotic cells were expressed as the average number per square micrometre. 


\section{HPLC determination of genistein}

Gen was measured using HPLC (Type 168; Beckman, Miami, FL, USA) assay. Approximately, $1 \mathrm{~g}$ of brain tissue from different regions was weighed and homogenised in $5 \mathrm{ml}$ of saline. Then, the homogenates from different brain regions were pooled by treatment. The samples $(1 \mathrm{ml}$ for brain tissue and $0.5 \mathrm{ml}$ for serum) were added to $0.2 \mathrm{ml}$ of an internal standard solution and $1.5 \mathrm{ml}$ of an extraction solution (methyl-tert-butyl ether-normal pentane, 8:2 v/v) before being vortexed for $2 \mathrm{~min}$. After centrifugation ( $3000 \mathrm{~g}$, $5 \mathrm{~min}$ ), each supernatant was transferred to a clean tube. The supernatant was extracted twice, and the two collections were pooled for drying under vacuum at ambient temperature overnight. The residue was reconstituted in $50 \mu \mathrm{l}$ of methanol, and a total of $20 \mu$ l of each sample was added to the column for HPLC analysis.

Primary standards $(100 \mu \mathrm{g} / \mathrm{ml})$ were prepared in methanol. Working standards $(0.5,1,5,10,20$ and $30 \mu \mathrm{g} / \mathrm{ml})$ were prepared in $0.1 \mathrm{ml}$ methanol-water $(80: 20, \mathrm{v} / \mathrm{v})$ before being dried under vacuum. Samples and standards were loaded onto a C8 column $(5 \mu \mathrm{m}, 250 \times 4.6 \mathrm{~mm}$; Phenomenex, Torrance, CA, USA) with a mobile phase of sodium citrate buffer $(\mathrm{pH} 4 \cdot 1,50 \mathrm{mmol} / \mathrm{l})$-acetonitrile $(6: 4, \mathrm{v} / \mathrm{v})$ at a flow rate of $1 \mathrm{ml} / \mathrm{min}$. The limit of quantitation for Gen was $12 \mathrm{ng} / \mathrm{ml}$.

The data are presented as the mean of separate determinations from two different experiments.

\section{Statistical analysis}

The effect of diet treatment was assessed by ANOVA with Statistical Package for the Social Sciences software (IBM, Beijing, People's Republic of China). Values are presented as means and standard deviations. If significant differences were observed, the least significant difference $t$ test was applied to the multiple comparisons of means between each group.

\section{Results}

Effects of genistein on cognitive abilities of ovariectomised rats

In the visual spatial learning and memory test, the average escape latency declined rapidly during the five consecutive days. Although there was a trend of decline of the escape latency in the E2 and Gen-L groups on days 2, 3 and 5 than in the Ovx group, there were still no significant differences among the population means of the five groups at any time point (Table 1).

In the spatial probe trial test, the memory frequency on the original platform quadrant and the swimming time taken to find the original position of the platform were determined. The escape latency was enhanced, while the memory frequency was reduced in the Ovx group than in the sham group. The declined cognitive abilities after ovariectomy were significantly improved in the E2 and Gen-L groups as exhibited by reduced escape latency and increased memory frequency. Neither of the two parameters was improved in the Gen-H group (Table 1).
Table 1. Average escape latency in the spatial learning and memory test and swimming and memory frequency in the spatial probe trial test of rats under different treatments $(n 13)$

(Mean values and standard deviations)

\begin{tabular}{|c|c|c|c|c|c|c|}
\hline \multirow[b]{2}{*}{ Groups } & \multicolumn{2}{|c|}{$\begin{array}{l}\text { Escape } \\
\text { latency (s) }\end{array}$} & \multicolumn{2}{|c|}{$\begin{array}{l}\text { Swimming } \\
\text { time (s) }\end{array}$} & \multicolumn{2}{|c|}{$\begin{array}{c}\text { Memory } \\
\text { frequency (s) }\end{array}$} \\
\hline & Mean & SD & Mean & SD & Mean & SD \\
\hline Sham & 43.97 & $28 \cdot 61$ & $51 \cdot 7$ & $36 \cdot 8$ & 24.9 & 4.5 \\
\hline Ovx & 56.85 & 28.71 & $88.9^{\star}$ & 35.1 & $18 \cdot 7^{*}$ & $2 \cdot 7$ \\
\hline E2 & 46.69 & 29.05 & $57.3 \dagger$ & 21.7 & $25.5+\dagger$ & 5.4 \\
\hline Gen-L & 48.45 & $27 \cdot 19$ & $70.4 \dagger$ & 13.8 & $23.6 \dagger$ & 5.4 \\
\hline Gen-H & 49.45 & 27.45 & $78 \cdot 1^{*}$ & 25.5 & 22.7 & $2 \cdot 1$ \\
\hline
\end{tabular}

Ovx, ovariectomised; E2, 17ß-oestradiol; Gen-L, genistein-low; Gen-H, genistein-high Mean values were significantly different from the sham group: ${ }^{\star} P<0.05$.

Mean values were significantly different from the Ovx group: $\dagger P<0.05, \dagger \dagger P<0.01$.

\section{Acetylcholinesterase activity}

AchE activity was significantly higher in the Ovx group than in the sham group $(P=0.007$ in the hippocampus and $P=0.043$ in the cortex), and was remarkably suppressed by either E2 or Gen treatment. To our surprise, AchE activity was suppressed by Gen so profoundly $(P=0.006$ in the hippocampus, $P=0.008$ in the cortex $v$. Ovx) that the magnitude of suppression was even higher than that observed in the E2 group (Fig. 1).

\section{Effects of genistein on brain superoxide dismutase, malondialdehyde and monoamine oxidases}

To test the degree of oxidative damages, brain SOD, MDA and MAO were measured (Table 2). In the hippocampus, there were significant differences of SOD level among the five groups $(P=0 \cdot 043)$, while in the cortex, no differences were found. It was further demonstrated by least significant difference $t$ test that there was a sharp decline of hippocampus SOD in the Ovx group than in the sham controls, which could be recovered by $\mathrm{E} 2$ or Gen-L treatment (Table 2).

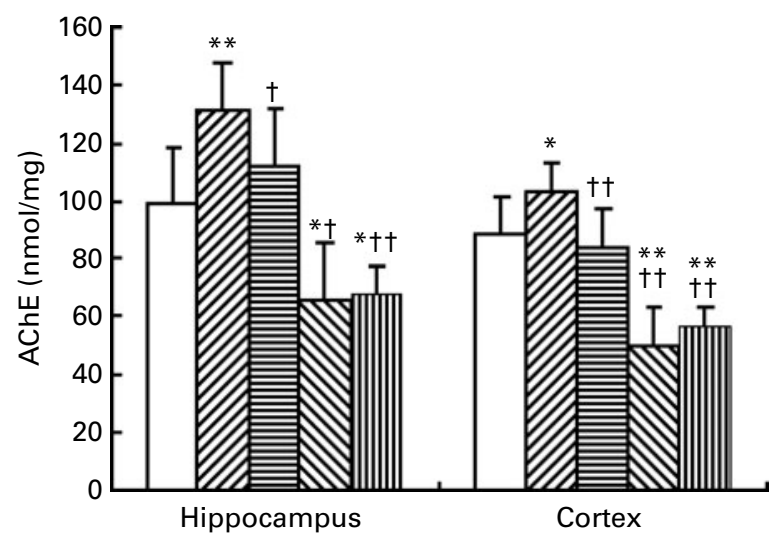

Fig. 1. Acetylcholinesterase (AchE) activities in the frontal cortex and hippocampus of rats orally administered with $17 \beta$-oestradiol (E2), $15 \mathrm{mg} / \mathrm{kg}$ genistein $($ Gen-low $(L))$ and $30 \mathrm{mg} / \mathrm{kg}($ Gen-high $(H))$ for 6 weeks $(n 5)$. Values are means and standard deviations. Mean values were significantly different when compared with the sham group: ${ }^{\star} P<0.05$, ${ }^{* *} P<0.01$. Mean values were significantly different when compared with the ovariectomised (Ovx) group: $\dagger P<0.05, \dagger+P<0.01$. $\square$, Sham; 四, Ovx; 目, E2; $\mathbb{8}$, Gen-L; m, Gen-H. 
Table 2. Mitochondria-generated superoxide dismutase (SOD), monoamine oxidases (MAO) and malondialdehyde (MDA) in the frontal cortex and hippocampus of rats under different treatments $(n 5)$

(Mean values and standard deviations)

\begin{tabular}{|c|c|c|c|c|c|c|c|c|c|c|c|c|}
\hline \multirow[b]{3}{*}{ Groups } & \multicolumn{4}{|c|}{ SOD (U/mg) } & \multicolumn{4}{|c|}{ MDA (nmol/mg) } & \multicolumn{4}{|c|}{$\mathrm{MAO}(\mathrm{nmol} / \mathrm{mg})$} \\
\hline & \multicolumn{2}{|c|}{ Hippocampus } & \multicolumn{2}{|c|}{ Cortex } & \multicolumn{2}{|c|}{ Hippocampus } & \multicolumn{2}{|c|}{ Cortex } & \multicolumn{2}{|c|}{ Hippocampus } & \multicolumn{2}{|c|}{ Cortex } \\
\hline & Mean & SD & Mean & SD & Mean & SD & Mean & SD & Mean & SD & Mean & SD \\
\hline Sham & $3014 \cdot 4$ & $1067 \cdot 4$ & $2566 \cdot 9$ & $661 \cdot 3$ & $108 \cdot 2$ & $29 \cdot 3$ & 135.9 & $21 \cdot 1$ & $152 \cdot 8$ & $39 \cdot 2$ & $144 \cdot 9$ & $102 \cdot 2$ \\
\hline Ovx & $1884 \cdot 2^{*}$ & $370 \cdot 8$ & 1923.5 & $591 \cdot 2$ & $230 \cdot 7^{\star \star}$ & $83 \cdot 3$ & $220 \cdot 4^{*}$ & $55 \cdot 7$ & $223 \cdot 0^{*}$ & $66 \cdot 8$ & $165 \cdot 2$ & $59 \cdot 8$ \\
\hline E2 & $2778.6 \dagger$ & $150 \cdot 7$ & $2054 \cdot 6$ & 239.8 & $137 \cdot 7 \dagger$ & $14 \cdot 7$ & $124 \cdot 1 \dagger \dagger$ & $26 \cdot 9$ & $134.2 † \dagger$ & $46 \cdot 6$ & $99.5+\dagger$ & $18 \cdot 3$ \\
\hline Gen-L & $2772 \cdot 3 \dagger$ & 1155.4 & $2023 \cdot 7$ & 255.5 & $216 \cdot 9^{\star} \ddagger$ & $58 \cdot 7$ & 148.0† & 36.9 & 188.8 & $34 \cdot 2$ & $106 \cdot 1 \dagger$ & $22 \cdot 4$ \\
\hline Gen-H & 2398.9 & 294.3 & 2234.6 & $653 \cdot 8$ & $203 \cdot 3^{\star \star} \ddagger$ & 34.9 & $181 \cdot 7$ & $92 \cdot 1$ & $163.8 \dagger$ & $10 \cdot 9$ & $114.4 \dagger$ & 30.6 \\
\hline
\end{tabular}

Ovx, ovariectomised; E2, 17ß-oestradiol; Gen-L, genistein-low; Gen-H, genistein-high.

Mean values were significantly different from the sham group: ${ }^{*} P<0.05,{ }^{* \star} P<0.01$.

Mean values were significantly different from the Ovx group: $\dagger P<0.05$, $\dagger \dagger P<0.01$.

$¥$ Mean values were significantly different from the E2 group $(P<0.05)$.

(a)
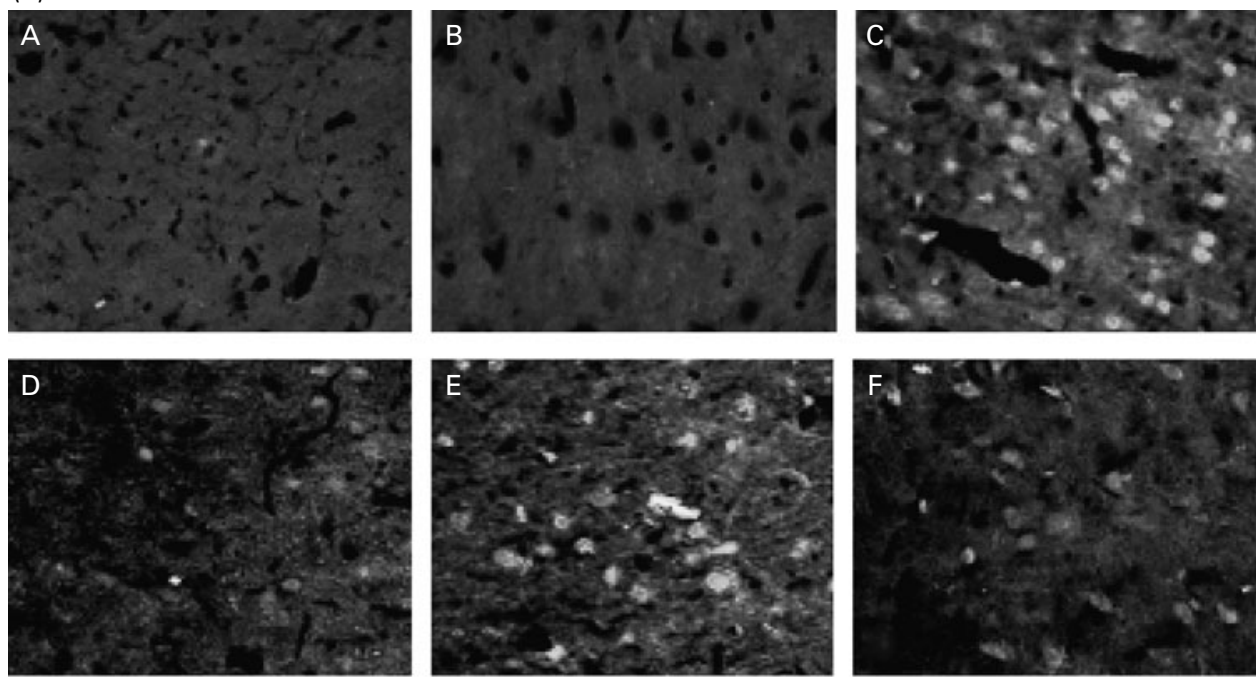

(b)
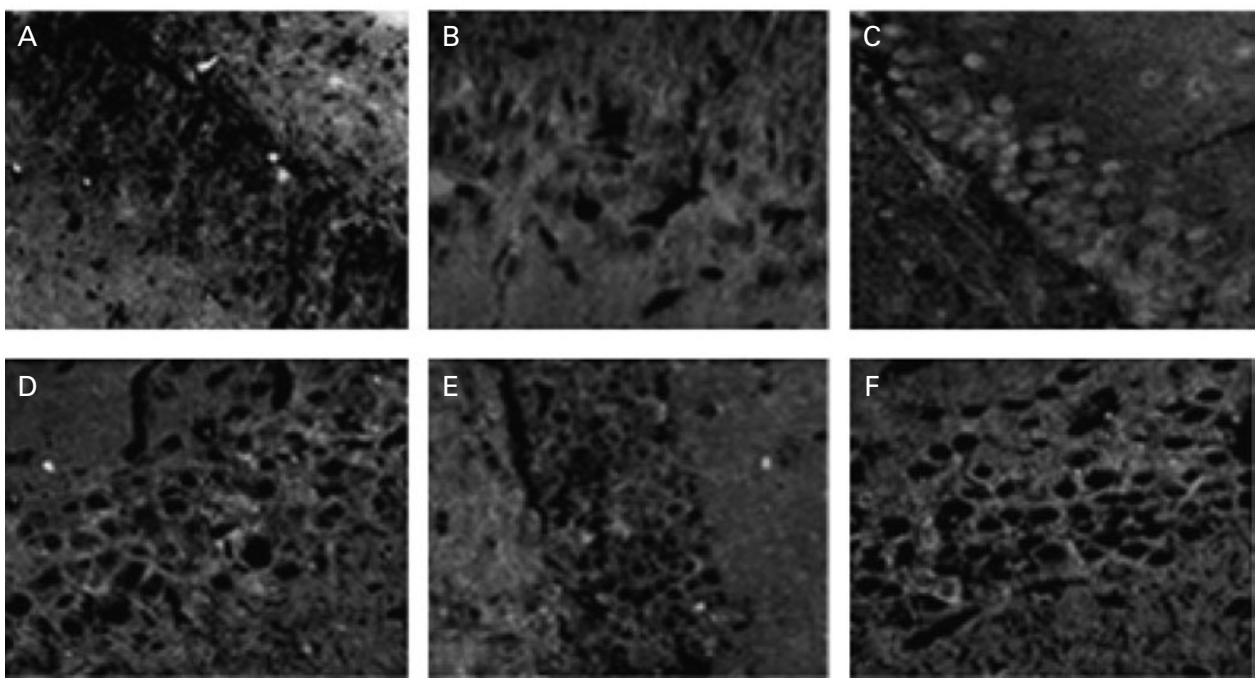

Fig. 2. Representative micrographs of TUNEL-positive cells in the frontal cortex (a) and hippocampal CA1 region (b) from negative control (A), sham $(B)$, ovariectomised $(C), 17 \beta$-oestradiol $(D)$, genistein-low $(E)$ and genistein-high $(F)$ rats $(n 8)$. 
It was noteworthy that $30 \mathrm{mg} / \mathrm{kg}$ Gen could not exert the same effect as $15 \mathrm{mg} / \mathrm{kg}$ Gen, suggesting a dose-dependent regulation of SOD by Gen.

In both the hippocampus and cortex, MDA was remarkably higher in the Ovx group than in the sham controls $(P=0.004$ in the hippocampus and $P=0.031$ in the cortex). E2 could attenuate the elevated MDA levels in both of the regions $(P=0.008$ in the hippocampus and $P=0.007$ in the cortex $v$. Ovx). On the other hand, neither dose of Gen could affect the elevated MDA levels in the hippocampus, but in the cortex, MDA level was reduced by the low dosage of Gen $(P=0 \cdot 005)$ (Table 2).

MAO was elevated after ovariectomy in the hippocampus, and it was recovered at a level that was similar to that observed in the sham controls by both E2 and high dose of Gen (Table 2). In the cortex, although MAO was not altered by ovariectomy, it could be suppressed by both E2 and Gen.

\section{Effects of genistein on the apoptosis of the brain}

Caspase-3 activity in the hippocampus region was increased in the Ovx group $(247.24(\mathrm{SD} 27.50) \mathrm{pmol} / \mathrm{mg})$ than in the sham group (102.53 (SD 39.86) $\mathrm{pmol} / \mathrm{mg}, P=0.003)$. Treatment with low dose and high dose of Gen could inhibit caspase-3 activity to 157.36 (SD 29.97) and 144.99 (SD 27.91) pmol/mg, respectively $(P=0 \cdot 036)$. Supplementation with E2 could decrease caspase-3 activity to even lower levels (93.87 (SD 16.78) pmol/ mg, $P=0 \cdot 002$ ).

In line with caspase- 3 , in both the hippocampus and cortex, the apoptotic cells as detected by TUNEL staining were notably enhanced in the Ovx group than in the sham controls (Fig. 2(a) and (b)). Replacement of Gen and E2 could reduce the number of the apoptotic cells prominently in both the hippocampus and cortex to almost a similar extent (Fig. 2 and Table 3).

\section{Genistein concentrations}

Gen concentrations in both sham and Ovx groups were too low to be detected, while the levels of Gen in both the brain and serum were still at the physiological range (Table 4). Gen levels in the Gen-H group that were even lower than

Table 3. The number of terminal deoxynucleotidyl transferase dUTP nick end labelling-positive cells per square micrometre in the brain $(n 8)$

(Mean values and standard deviations)

\begin{tabular}{|c|c|c|c|c|}
\hline \multirow[b]{2}{*}{ Groups } & \multicolumn{2}{|c|}{ Hippocampus } & \multicolumn{2}{|c|}{ Frontal cortex } \\
\hline & Mean & SD & Mean & SD \\
\hline Sham & $1 \cdot 8$ & 0.37 & 1.4 & 0.2 \\
\hline Ovx & $32 \cdot 4^{\star}$ & 1.4 & $28 \cdot 8^{\star}$ & $1 \cdot 3$ \\
\hline E2 & $11.4^{*} \dagger$ & 0.5 & $14 \cdot 0^{*} \dagger$ & 0.7 \\
\hline Gen-L & $14 \cdot 2^{*} \dagger$ & 0.9 & $17 \cdot 0^{\star} † \ddagger \ddagger$ & 0.7 \\
\hline Gen-H & $13 \cdot 6^{*} \dagger$ & $1 \cdot 2$ & $18 \cdot 6^{\star} † \ddagger \ddagger$ & 0.8 \\
\hline
\end{tabular}

Ovx, ovariectomised; E2, 17ß-oestradiol; Gen-L, genistein-low; Gen-H, genistein-high.

* Mean values were significantly different from the sham group $(P<0.05)$ † Mean values were significantly different from the Ovx group $(P<0.05)$. 扫 Mean values were significantly different from the E2 group $(P<0.01)$.
Table 4. Genistein concentrations in the brain and serum determined by HPLC in ovariectomised rats after oral administration with genistein for 6 weeks (pooled by treatment, $n 5$ per group)

(Mean values and standard deviations)

\begin{tabular}{llllll}
\hline & \multicolumn{2}{c}{ Brain $(\mathrm{ng} / \mathrm{g})$} & & \multicolumn{2}{c}{ Serum $(\mathrm{nmol} / \mathrm{l})$} \\
\cline { 2 - 3 } Groups & Mean & SD & & Mean & SD \\
\hline Gen-L & 96.7 & 18.7 & & 257.5 & $21 \cdot 1$ \\
Gen-H & 74.3 & 21.8 & & $190 \cdot 1$ & 23.3 \\
\hline
\end{tabular}

Gen-L, genistein-low; Gen-H, genistein-high.

those in the Gen-L group and serum concentration that was almost three times of that in the tissue were of particular interest.

\section{Discussion}

In the present study, we explored the novel antioxidant mechanism of Gen on neuroprotection in Ovx rats. Our data revealed that Gen could inhibit the neurodegeneration induced by ovariectomy via modulation of mitochondrial oxidative stress.

Gen that was orally administered at $15 \mathrm{mg} / \mathrm{kg}$ instead of at $30 \mathrm{mg} / \mathrm{kg}$ for 6 weeks was able to reduce the escape latency and to increase the memory frequency, which were compromised by ovariectomy. In the visual spatial learning and memory test, although the escape latency showed no difference among the five groups, there was still a trend of decline in the E2 and Gen-L groups. It was not surprising that in $\mathrm{Xu}$ et al. study $^{(8)}$, the average escape latency was significantly reduced by Gen that was injected subcutaneously at about $10 \mathrm{mg} / \mathrm{kg}$ every other day for 5 weeks. This discrepancy might be due to Gen being purchased from different companies or the disparate injection routes used, subcutaneously in $\mathrm{Xu}$ et al. study and through oral administration in our rats, both of which might result in differential Gen levels. The absence of improvement in the learning abilities in the Gen$\mathrm{H}$ group was not unexpected. Considering the non-linear pharmacokinetics of Gen in the gastrointestinal tract due to its low water solubility and transportation saturability, the concentrations of Gen in both the serum and brain of the Gen-H rats were even lower than those in the Gen-L rats in the present study, which thus might be less biologically effective.

It was reported previously that the rats fed on soya isoflavone diet took significantly shorter time for swimming than the group fed on the control diet in spatial delayed matching-to-place performance ${ }^{(14)}$. Moreover, isoflavone supplementation in healthy males may also enhance cognitive processes $^{(15)}$. Gen is only one of the six constituents of isoflavone, thus it may be less effective than that given in combination $^{(9)}$. In a double-blind randomised trial, the use of soya protein containing isoflavones did not improve cognitive function in healthy women when it was administered at the age of 60 years or later ${ }^{(16)}$. This implies that the effect of Gen may be influenced by the timing of administration. In the present study, Gen was given 1 week after the surgery, while in Xu's study ${ }^{(8)}$, it was given from the second day after the surgery. The later the administration of the Gen is done, the less is the effect it exerts. 
In the spatial probe trial test, Gen did exert a beneficial effect. The memory frequency was reduced and the swimming time was prolonged in the Ovx group, both of which were reversed by Gen-L treatment. The present study along with $\mathrm{Xu}^{\prime}$ s study ${ }^{(8)}$ suggested that Gen at a certain dose was able to improve the memory abilities in rats.

It is intriguing that the memory abilities were only improved in the Gen-L group but not in the Gen-H group. The effect of phyto-oestrogen was reported to differ depending on the dose and the sex. In the delayed matching-toplace water maze task, the swimming time was shortened by use of low levels of isoflavones $(0 \cdot 3 \mathrm{~g} / \mathrm{kg})$, but no change was found when higher levels of isoflavones were used $(1.2 \mathrm{~g} / \mathrm{kg})^{(14)}$. This may be explained by the fact that Gen at a high dose may induce cytotoxicity and apoptosis in the brain $^{(17,18)}$, and could increase the levels of cleaved caspase- 3 in vivo and in primary neuronal culture ${ }^{(17)}$.

Besides the lower concentrations of Gen in the Gen-H group accounting for the less efficiency of higher dose Gen, there might be other possibilities. In our previous study, the percentages of serum Gen $v$. total Gen (Gen plus glucuronidated Gen) after intake of different doses of Gen were $18.68 \%$ for $12.5 \mathrm{mg} / \mathrm{kg}$ and $4.16 \%$ for $50 \mathrm{mg} / \mathrm{kg}$, respectively. Despite the total Gen levels being still higher at $50 \mathrm{mg} / \mathrm{kg}$ dose than at $12.5 \mathrm{mg} / \mathrm{kg}$ dose, the conjugated form might not be an effective antioxidant as Gen.

It was reported that isoflavones are ingested mainly in the glycoside form, and undergo extensive hydrolysis by intestinal and bacterial $\beta$-glucosidases that release the principal aglycones, Gen and daidzein. Following absorption in the gastrointestinal tract, isoflavones are conjugated with glucuronic acid or sulphuric acid by the enzymes in the intestinal epithelium and the liver ${ }^{(19)}$. The formation of conjugates converts isoflavones to more water-soluble products, and may affect their chemical and biological activities. For example, the gut and liver metabolite equol can be a more potent antioxidant than its parent isoflavone daidzein. However, sulphation, which masks the important hydroxyl groups of the isoflavone molecules, could decrease the antioxidant potential of these compounds ${ }^{(20)}$. Since most of the Gen circulates as a conjugate at a high-dose intake, the glucuronidated Gen might not be an effective antioxidant in vivo.

To further investigate the neurotransmitters that account for the cognitive improvement by Gen, AchE activity was examined in the brain. Cholinergic parameters decline continuously during ageing in the hippocampus, cortex and other areas related to recognition ${ }^{(18)}$. In the present study, the rats subjected to ovariectomy presented a significant increase in AchE activity, which was similar to that reported previously ${ }^{(21)}$. For the first time, we have also observed the in vivo inhibitory effect of Gen on AchE activity, which was consistent with that found in soya isoflavones ${ }^{(14)}$, suggesting that Gen could ameliorate deficits in memory tasks resulting from the loss of cholinergic input or cholinergic degeneration in Ovx rats.

Recent evidence has indicated that phyto-oestrogen may protect against the neurodegeneration via maintenance of mitochondrial structure and functions ${ }^{(22)}$. This prompted us to examine the key parameters of mitochondrial oxidative stress after Gen treatment.

First, there were significant differences in SOD content in the hippocampus, but not in the cortex, among the five groups. This suggests that modulation of brain SOD by Gen may be region dependent. E2 or Gen-L treatment, but not Gen-H treatment, could recover the decline of SOD by Ovx (Table 2), suggesting that modulation of brain SOD by Gen was also dose dependent. It was reported previously that Gen could result in a relatively higher SOD level in cultured cortical neurons ${ }^{(23)}$ and reverse the decreased serum SOD in Ovx rats ${ }^{(8)}$, both of which reflect the effect of Gen on brain SOD indirectly. For the first time, we have also reported SOD content in the tissue from two regions of the brain, which could reveal the neuroprotective effect of Gen more precisely.

Secondly, MDA and MAO levels could be enhanced after ovariectomy, which were further suppressed by E2. The increased MDA level was only inhibited in the cortex in the Gen-L group, suggesting a region- and dose-dependent effect of Gen. In the cerebral injury model of ischaemia/reperfusion, Gen was shown to decrease reactive oxygen species generation, MDA concentration and the apoptotic indices in rat hippocampus $^{(24)}$. The oxidative damage in this model was more severe than that in the Ovx rats, and the hippocampus was more inclined to be insulted. Our findings of MDA modulation in the brain tissue were supportive of those of Xu's study ${ }^{(8)}$, in which Gen could reduce the serum MDA distinctly.

Lastly, we found that Gen could suppress MAO activity in the brain. In the cortex, MAO was remarkably reduced by Gen at both doses, but in the hippocampus, it was only decreased in the Gen-H group but not in the Gen-L group. The dose independency of MAO inhibition added more complexity to the action of Gen in the brain. Monoaminergic signalling is regarded as one of the key mechanisms for the modulation of mood and emotion, as well as for the control of motor, perceptual and cognitive functions ${ }^{(3)}$. Attenuation of the oxidative stress by the inactivation of MAO may contribute to enhanced cognitive ability by Gen.

Significant attenuation of mitochondrial stress by Gen may result in less apoptosis being induced by ovariectomy. In cultured cortical neurons, Gen was observed to down-regulate caspase- 3 expression and lead to fewer apoptotic cells ${ }^{(25)}$. We found that Gen treatment not only decreased the number of TUNEL-positive cells, but also reduced the level of caspase-3 activity in the brain, indicating that the neuroprotection of Gen is through the inhibition of the mitochondria-mediated apoptotic pathway.

Other mechanisms may also account for the enhanced memory induced by Gen. The activation of cyclic AMPresponse element-binding protein-dependent gene expression is thought to be critical for the formation of different types of long-term memory ${ }^{(23)}$. As Gen was reported to induce cyclic AMP-response element-binding protein phosphorylation in the medial preoptic area and anteroventral periventricular nucleus $^{(24)}$, it is possible that Gen also exerts its effect via the activation of cyclic AMP-response element-binding protein in the present study. Besides the activation of transcription factors described above, phyto-oestrogen could also bind to oestrogen receptor- $\beta$ to exhibit its effect on neuronal survival against toxic insults and promote neural proactive defence mechanisms against neurodegeneration ${ }^{(25)}$. In summary, Gen exerted its neuroprotective effect via multiple pathways, one of which was by modulation of the mitochondrial oxidative stress, a newly emerging concept that may lead to in-depth understanding of its biological characteristics. 
The present study systemically demonstrated the antioxidant activities of Gen in different brain regions and its ultimate consequence on neurodegeneration in rats, which could be extrapolated to women after menopause; nevertheless, the prescription of Gen for long-term use should be carefully considered $^{(8)}$.

\section{Acknowledgements}

This work was supported by Chinese National Natural Science Foundation (no. 30973120). The authors have no conflict of interest to declare. Y.-H. H. designed and conducted the study. Q.-H. Z. initiated the experiments and wrote the manuscript.

\section{References}

1. Simpkins JW, Yi KD \& Yang SH (2009) Role of protein phosphatases and mitochondria in the neuroprotective effects of estrogens. Front Neuroendocrinol 30, 93-105.

2. Jayakumar T, Thomas PA \& Geraldine P (2007) Protective effect of an extract of the oyster mushroom, Pleurotus ostreatus, on antioxidants of major organs of aged rats. Exp Gerontol 42, 183-191.

3. Bortolato M, Chen K \& Shih JC (2008) Monoamine oxidase inactivation: from pathophysiology to therapeutics. Adv Drug Deliv Rev 60, 1527-1533.

4. Borras C, Sastre J, Garcia-Sala D, et al. (2003) Mitochondria from females exhibit higher antioxidant gene expression and lower oxidative damage than males. Free Radic Biol Med 34, 546-552.

5. Guevara-Guzman R, Arriaga V, Kendrick KM, et al. (2009) Estradiol prevents ozone-induced increases in brain lipid peroxidation and impaired social recognition memory in female rats. Neuroscience 159, 940-950.

6. Razmara A, Duckles SP, Krause DN, et al. (2007) Estrogen suppresses brain mitochondrial oxidative stress in female and male rats. Brain Res 1176, 71-81.

7. Simpkins JW, Yi KD, Yang SH, et al. (2009) Mitochondrial mechanisms of estrogen neuroprotection. Biochim Biophys Acta (Epublication ahead of print version).

8. Xu J, Zhu J, Shi C, et al. (2007) Effects of genistein on hippocampal neurodegeneration of ovariectomized rats. $J \mathrm{Mol}$ Neurosci 31, 101-112.

9. Zhao L, Mao Z \& Brinton RD (2009) A select combination of clinically relevant phytoestrogens enhances estrogen receptor beta-binding selectivity and neuroprotective activities in vitro and in vivo. Endocrinology 150, 770-783.

10. Borras C, Gambini J, Lopez-Grueso R, et al. (2010) Direct antioxidant and protective effect of estradiol on isolated mitochondria. Biochim Biophys Acta 1802, 205-211.
11. Azadbakht L, Kimiagar M, Mehrabi Y, et al. (2007) Dietary soya intake alters plasma antioxidant status and lipid peroxidation in postmenopausal women with the metabolic syndrome. Br J Nutr 98, 807-813.

12. Irwin RW, Yao J, Hamilton RT, et al. (2008) Progesterone and estrogen regulate oxidative metabolism in brain mitochondria. Endocrinology 149, 3167-3175.

13. Enari M, Sakahira H, Yokoyama H, et al. (1998) A caspaseactivated DNase that degrades DNA during apoptosis, and its inhibitor ICAD. Nature 391, 43-50.

14. Lee YB, Lee HJ, Won MH, et al. (2004) Soy isoflavones improve spatial delayed matching-to-place performance and reduce cholinergic neuron loss in elderly male rats. $J$ Nutr 134, 1827-1831.

15. Thorp AA, Sinn N, Buckley JD, et al. (2009) Soya isoflavone supplementation enhances spatial working memory in men. Br J Nutr 102, 1348-1354.

16. Kreijkamp-Kaspers S, Kok L, Grobbee DE, et al. (2004) Effect of soy protein containing isoflavones on cognitive function, bone mineral density, and plasma lipids in postmenopausal women: a randomized controlled trial. JAMA 292, 65-74.

17. Choi EJ \& Lee BH (2004) Evidence for genistein mediated cytotoxicity and apoptosis in rat brain. Life Sci 75, 499-509.

18. Jones DN, Barnes JC, Kirkby DL, et al. (1995) Age-associated impairments in a test of attention: evidence for involvement of cholinergic systems. J Neurosci 15, 7282-7292.

19. Rimbach G, Weinberg PD, de Pascual-Teresa S, et al. (2004) Sulfation of genistein alters its antioxidant properties and its effect on platelet aggregation and monocyte and endothelial function. Biochim Biophys Acta 1670, 229-237.

20. Turner R, Baron T, Wolffram S, et al. (2004) Effect of circulating forms of soy isoflavones on the oxidation of low density lipoprotein. Free Radic Res 38, 209-216.

21. Monteiro SC, Matte C, Delwing D, et al. (2005) Ovariectomy increases $\mathrm{Na}^{+}, \mathrm{K}^{+}$-ATPase, acetylcholinesterase and catalase in rat hippocampus. Mol Cell Endocrinol 236, 9-16.

22. Xu XW, Shi C, He ZQ, et al. (2008) Effects of phytoestrogen on mitochondrial structure and function of hippocampal CA1 region of ovariectomized rats. Cell Mol Neurobiol 28, 875-886.

23. Ho KP, Li L, Zhao L, et al. (2003) Genistein protects primary cortical neurons from iron-induced lipid peroxidation. Mol Cell Biochem 247, 219-222.

24. Liang HW, Qiu SF, Shen J, et al. (2008) Genistein attenuates oxidative stress and neuronal damage following transient global cerebral ischemia in rat hippocampus. Neurosci Lett 438, 116-120.

25. Yu HL, Li L, Zhang XH, et al. (2009) Neuroprotective effects of genistein and folic acid on apoptosis of rat cultured cortical neurons induced by beta-amyloid 31-35. Br J Nutr 102, $655-662$. 